\title{
Atomic-level structural identification for prediction of localized shear deformation in metallic glasses
}

\author{
Ronghao Shi ${ }^{\mathrm{a}, \mathrm{b}}$, Pan Xiao ${ }^{\mathrm{a}, *}$, Rong Yang ${ }^{\mathrm{a}}$, Yilong Bai ${ }^{\mathrm{a}}$ \\ a LNM, Institute of Mechanics, Chinese Academy of Sciences, Beijing 100190, China \\ ${ }^{\mathrm{b}}$ School of Engineering Sciences, University of Chinese Academy of Sciences, Beijing 100049, China
}

\section{A R T I C L E I N F O}

\section{Article history:}

Received 8 October 2019

Revised 19 December 2019

Accepted 13 January 2020

Available online 15 January 2020

\section{Keywords:}

Metallic glasses

Shear transformation zones

Nonaffine displacement

Molecular simulations

Dynamical matrix

\begin{abstract}
A B S T R A C T
Unlike obviously topological defects in crystals, "defect" identification in metallic glasses (MGs) is still controversial and under investigation. Based on molecular simulations and analysis of MG samples, a structural parameter related to the Laplacian of local equivalent stiffness is proposed for prediction of localized shear deformation in MGs. It is found that local regions with the parameter of positive and relatively large value represent several particular modes of "stiffness valleys" in initial configuration which will strengthen local shear deformation and lead to the formation of shear transformation zones (STZs). More than $80 \%$ of the locations where STZs are formed in six types of MG samples under athermal quasistatic shear deformation match well with the regions predicted by the parameter calculated from initial configuration of the samples. The parameter not only reveals the relationship among local heterogeneity, nonaffine displacement, and shear localization, but also provides an efficient way for predicting the activation of STZs in MGs.
\end{abstract}

(c) 2020 Elsevier Ltd. All rights reserved.

\section{Introduction}

Defects of crystals are obvious for their unique topological structures (Taylor, 1934), while "defects" of metallic glasses (MGs) and their plastic behaviors are further obscure. Relentless research has paved the way for these fundamental issues during the past decades by experiments, physical models and computational simulations (Argon, 1979; Ding et al., 2014a, 2014b; Jiang et al., 2010; Jiang and Dai, 2010; Maloney and Lemaître, 2006; Spaepen, 1977; Tian et al., 2017; Yang et al., 2016). Though a well-defined "defect" is not clear in metallic glasses yet, activation of shear transformation zones (STZ) is regarded as the most critical plastic event (Argon, 1979) and a kind of "flow defect" (Schuh et al., 2007). The percolation of these events eventually results in shear band formation (Greer et al., 2013). Nevertheless, the structural origin of STZs is far from explicit (Wang and Wang, 2019).

Recently, soft spots and soft modes in MGs are reported to have strong correlation with the origin of STZs by plenty of molecular simulations and mathematical deduction (Ding et al., 2014a, 2014b; Fultz, 2010; Manning and Liu, 2011; Widmer-Cooper et al., 2008). By analyzing phonon modes derived from the dynamical matrix and local atomic packing structures in molecular simula-

\footnotetext{
* Corresponding author.

E-mail address: xiaopan@Inm.imech.ac.cn (P. Xiao).
}

tions, the soft spots are related to short-range order (SRO) including geometrically unfavored motifs (GUM) and local five-fold symmetry (L5FS) of MG in predicting STZ locations (Ding et al., 2014a, 2014b). However, defects identification based on soft spots is not so perfect as in crystals (Rottler et al., 2014) that several structure parameters were further proposed, like flexibility volume (Ding et al., 2016), inversion-symmetry order (Milkus and Zaccone, 2016), atomic bond length deviation (BLD) (Peng et al., 2017), orientational order (Yang et al., 2019), and softness (Cubuk et al., 2016).

Broadly speaking, these methods and parameters are kinds of representations of structural heterogeneity. Apparently, the lack of inversion symmetry in the local nearest-neighbor environment is intimately connected with nonaffinity (Zaccone, 2013; Zaccone and Scossa-Romano, 2011). Order parameters regarding the local non-centrosymmery were found strongly related to boson peak and nonaffine softening in glass and crystals (Milkus and Zaccone, 2016). The break of atomic level centrosymmetry leads to local heterogeneity of mechanical properties. Wang et al. discovered the percolation of STZs and shear band formation are dependent on the spatial heterogeneity of local shear moduli (Wang et al., 2018a, 2018b). Local shear relaxation events are found to be related to locally unstable eigenvalues, also referred as instantaneous normal modes (Palyulin et al., 2018; Zhang et al., 2019).

The local heterogeneity of mechanical properties then further induces special deformation behavior under external loadings. 
Specifically, nonaffine displacement driven by derivatives of local moduli (DiDonna and Lubensky, 2005) and Eshelby quadrupolar nonaffine displacement pattern around STZ (Bulatov and Argon, 1994; Hieronymus-Schmidt et al., 2017; Maloney and Lemaître, 2006) were observed by early research. However, the relationship between the local nonaffine displacement and the initial configuration of MG remains unclear. Recently, Xu et al. reported that the protocol-dependent behavior of STZ activation is governed by the stress gradient. They adopted activation-relaxation technique nouveau (ARTn) with a sacrifice of time efficiency to seek higher accuracy in defects identification in MG (Xu et al., 2018). These advances are promising and inspiring, but a simple universal quantified correlation of the heterogeneity and STZ activation is still a formidable challenge.

Here, we propose a distinct structural parameter which is related to the Laplacian and gradient of local equivalent stiffness in MG. This parameter could be a universal statistical parameter for "defects" identification in MGs. The parameter is also a kind of description of local heterogeneity, and its relation to nonaffine displacement and localized shear deformation is explained.

\section{Methods}

The mechanical analysis is based on results from molecular simulations on $\mathrm{Cu}_{64} \mathrm{Zr}_{36}$ MG systems with the embedded atom method (EAM) potentials (Cheng et al., 2009). Samples of five different sizes and six different components are covered in our simulations. The selected MG sample as shown in Fig. 1(a) is generated by first equilibrating a $\mathrm{Cu}_{64} \mathrm{Zr}_{36}$ atomic system at $2500 \mathrm{~K}$ for $2 \mathrm{~ns}$, and followed by rapid quenching $\left(10^{13} \mathrm{~K} / \mathrm{s}\right)$ to $1 \mathrm{~K}$ within NPT ensemble, during which the external pressure is held at zero. The radial distribution function (RDF) of the sample is analyzed to ensure its glass state (Ding et al., 2014a, 2014b). The prepared MG sample contains 9000 atoms with dimensions of about $6.0 \times 12.0 \times 2.0 \mathrm{~nm}$. Nowadays, simulations of a larger metallic glass system are not so difficult. Here we try to limit the dimension of our simulation box based on several considerations: (1) ensure that basic plastic events such as STZs and shear band nucleation can be observed; (2) try to use a quasi 2D simulation configuration, so a simplified 2D analysis can be performed and; (3) the condition of external loading can be transferred to local regions more effectively. Periodic boundary conditions are applied in all three dimensions. Athermal quasi-static (AQS) shear is conducted on the inherent structure by conjugate-gradient (CG) method (Maloney and Lemaittre, 2006). It is worth mentioning that results from both AQS and molecular dynamics (MD) in other works indicate that similar localized shear deformation occurs in MGs no matter whether there is temperature. Therefore, AQS simulation is performed here to get rid of noises caused by temperature as in MD simulations. At each loading step, a shear strain $(\gamma)$ of $0.01 \%$ is imposed on the system, and followed by energy minimization. The whole loading process includes 3000 steps with total $\gamma$ up to $30 \%$. The simulations are performed using the code LAMMPS (Plimpton, 1995) and molecular visualization is rendered using Ovito (Stukowski, 2010).

\section{Results and discussion}

\subsection{Shear deformation of MG sample}

The strain-stress curve in Fig. 1(e) indicates the sample deforms from elastic stage to shear flow state under the applied shear strain. The von Mises invariant ( $\eta^{\text {Mises }}$ ) (Shimizu et al., 2007) for each atom $\left(\eta_{i}{ }^{\text {Mises }}\right)$ is calculated to examine the location and evolution of STZs and shear bands at $\gamma=8 \%, 10 \%$ and $15 \%$ as shown in Fig. 1(b)-(d), respectively. Fig. 1(f) shows the accumulated $\eta_{i}^{\text {Mises }}$ of atoms averaged in slices at different $y$ coordinates $\left(\eta_{i}{ }^{\text {Mises }}\right)$. Apparently, for $y$ in range 5.0 to $10.0 \mathrm{~nm}$ where shear band is formed at the end, the variation of $\eta_{i}^{\text {Mises }}$ with $\gamma$ indicates that the evolution of STZs is history-dependent. In another words, the initial configuration of MG sample is related to the activation and evolu-
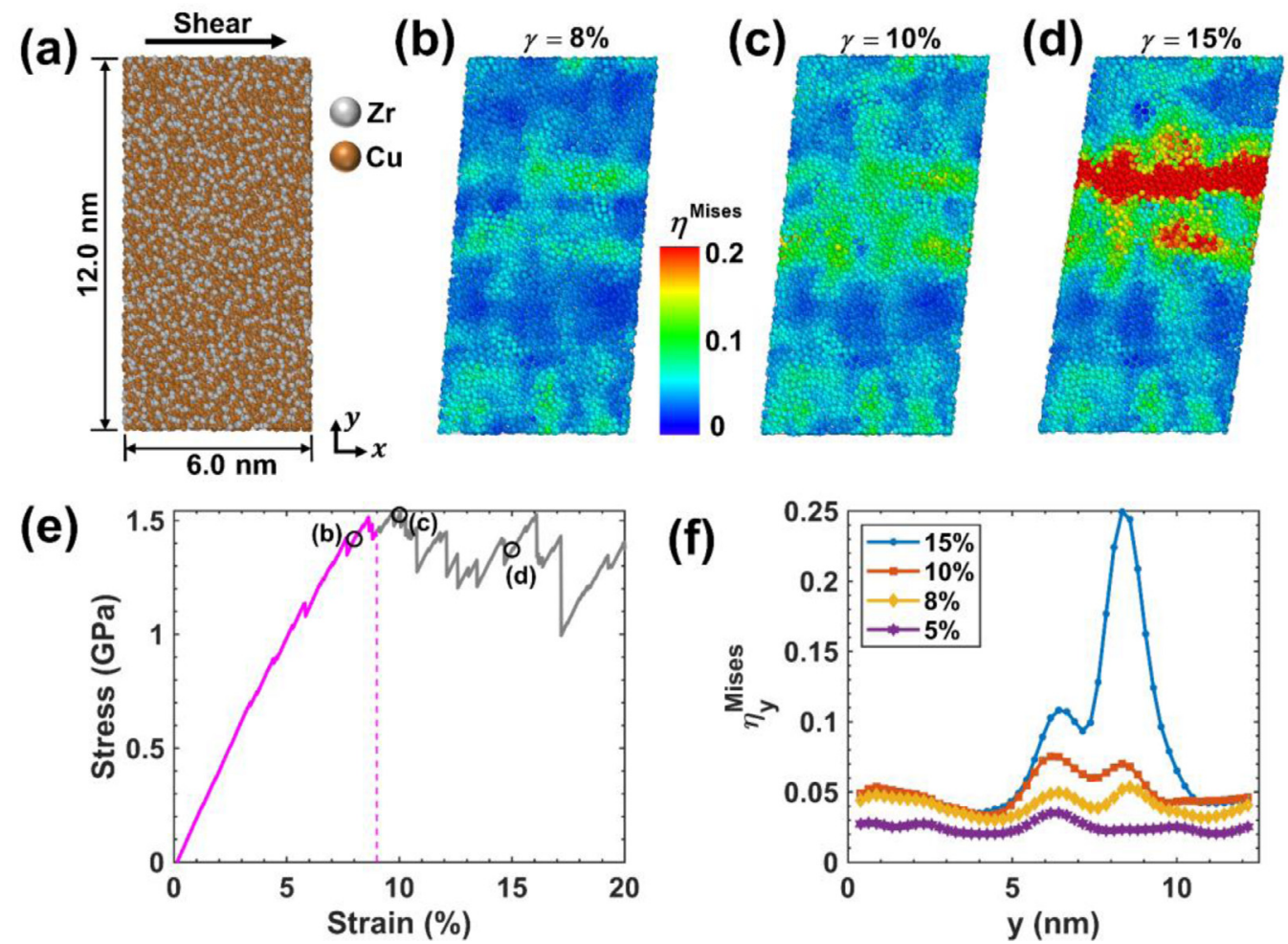

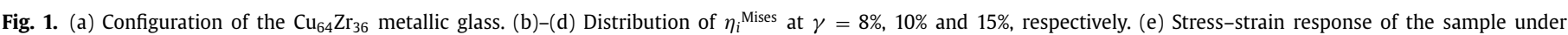
AQS loading condition. (f) Accumulated $\eta_{i}$ Mises of atoms averaged in slices at different $y$ coordinates. 
tion of STZs (Hu et al., 2016). Therefore, it is logical that there exist some "defects" in the initial configuration that would lead to the activation of STZs and finally to the formation of the shear band.

\subsection{Characterization of local equivalent stiffness}

Heterogeneity of the sample at its initial state is analyzed based on properties of its local dynamical matrix to identify the potential "defects" in the initial configuration. For a system with $N$ atoms, the $3 N \times 3 N$ full dynamical matrix $\left(D_{\text {full }}\right)$ is usually used to analyze vibrational modes of the systems as done in soft spots analysis (Ding et al., 2014a, 2014b; Manning and Liu, 2011; WidmerCooper et al., 2008). The local dynamical matrix for each atom (denoted by $D_{i}$ ) is a $3 \times 3$ matrix extracted from the diagonal of $D_{\text {full }}$ under local harmonic approximation (LeSar et al., 1989). Here the usage of $D_{i}$ instead of $D_{\text {full }}$ is due to several considerations. Firstly, $D_{i}$ is more convenient for local structural analysis than $D_{\text {full }}$. Operations of $D_{\text {full }}$ are usually related to global properties of an atomic system, e.g., frequency spectrum (Fultz, 2010), while $D_{i}$ is a local atomic quantity. Secondly, the computational cost of $D_{i}$ is much smaller than that of $D_{\text {full }}$. Specifically, the computational complexity of $D_{\text {full }}$ with $3 N \times 3 N$ elements is $O\left(N^{2}\right)$, while it is $O(N)$ for all $D_{i}$ with $N \times 3 \times 3$ elements. For example, in order to find a saddle point of an atom, ARTn has to determine $D_{\text {full }}$ and its eigenvalues many times (Wei et al., 2019). Thirdly, the physical meaning of $D_{i}$ is explicit. Eigenvalues of $D_{i}$ represent local vibrational frequencies of atom $i$. The determinant of $D_{i}\left(\left|D_{i}\right|\right)$, which is related to the product of the three eigenvalues, can be used as a measure to the local "stiffness" of atom $i$ in the cluster constructed with its neighbors. Here we refer the "stiffness" to a kind of equivalent stiffness for simplicity. Usually, $\left|D_{i}\right|$ is statistically proportional to the local density of atom $i$. The higher the local density is, the larger $\left|D_{i}\right|$ or stiffness will be. However, $\left|D_{i}\right|$ implies more than that, e.g. it includes the interactions between the atom and its neighbors and the atomic mass of different element. Moreover, $\left|D_{i}\right|$ can be utilized to express the Helmholtz free energy. Specifically, the Helmholtz free energy of an atomic system under the harmonic approximation can be given by (LeSar et al., 1989)

$F=V+k_{B} T \sum_{i=1}^{3 N} \ln \left[2 \sinh \left(\frac{h \omega_{i}}{2 k_{B} T}\right)\right]$

where the first term $V$ is potential energy, $\hbar$ is reduced Planck constant and $k_{B}$ is Boltzmann constant. The second term in Eq. (1) represents the energy from configurational and vibrational entropies. $\omega_{i}$ is the eigen frequency which can be determined by diagonalizing $D_{\text {full }}$. In the local harmonic approximation and classical limit, $2 \sinh \left(\frac{h \omega_{i}}{2 k_{B} T}\right) \sim \frac{h \omega_{i}}{k_{B} T}$ (LeSar et al., 1989), Eq. (1) can be further simplified to

$F=V+3 k_{B} T \sum_{i=1}^{N} \ln \frac{\hbar\left|D_{i}\right|^{1 / 6}}{k_{B} T}$,

where $\left|D_{i}\right|=\left(\omega_{1 i} \omega_{2 i} \omega_{3 i}\right)^{2}$ is the determinant of the local dynamical matrix of atom $i$. Eq. (2) has been successfully applied in molecular statistical thermodynamics (MST) method and several other multi-scale methods (Wang et al., 2008; Xiao et al., 2012) to characterize dislocations and phase transformations of nanostructures. It is worth noting that $\left|D_{i}\right|$ is related to the quartic term in a Taylor expansion of the interatomic potential and will be negative due to imaginary eigenfrequencies when local atomic configuration becomes unstable. According to the experience of applying Eq. (2) to investigate the mechanical behavior of different atomic systems (Wang et al., 2008; Xiao et al., 2012) at finite temperature by minimizing the Helmholtz free energy, imaginary eigenfrequencies appear only when local configuration becomes unstable, e.g. occurrence of dislocations or phase transformation. However, if $\left|D_{i}\right|$ is calculated only for the initial configuration at statistical stable state, there is no imaginary eigenfrequency. The dimensionless factor $D_{i}^{*}=\ln \left(\hbar\left|D_{i}\right|^{1 / 6} / k_{\mathrm{B}} T\right)$ in Eq. (2) represents part of the local entropy energy of atom $i$. The temperature $T$ in Eq. (2) is constant and set to be $1.0 \mathrm{~K}$ in the calculation to keep $D_{i}^{*}$ dimensionless and approximate the temperature close to $0 \mathrm{~K}$. Once temperature is given, only $\left|D_{i}\right|^{1 / 6}$ is variant in the analysis and others are constants. Therefore, it is physically meaningful to use $D_{i}^{*}$ for local heterogeneity analysis. Like many other atomic quantities used for structural analysis (Wei et al., 2019), whose critical length of distribution should approximate that of STZ (Zink et al., 2006), distribution of $D_{i}^{*}$ becomes distinct only when $D_{i}^{*}$ is averaged in a certain range around atom $i$. Here we refer $\bar{D}_{i}^{*}$ to the averaged $D_{i}^{*}$ with a specific averaging radius $\left(r_{\text {aver }}\right)$; and $r_{\text {aver }}$ is selected to be $1.0 \mathrm{~nm}$ which is around the fourth trough of RDF within which the interaction of surroundings is indispensable (Wei et al., 2019; Yang et al., 2016).

Although the field $\bar{D}^{*}$ reflects the distribution of local equivalent stiffness in the atomic system, neither $\bar{D}^{*}$ nor its gradient $\partial \bar{D}^{*}$ shows a significant or direct correlation with the activation of STZs. However, it is found that a product of the divergence and the absolute component difference of $\partial \bar{D}^{*}$ is spatially related with STZ atoms, as shown in Fig. 2(a). The combined parameter (denoted as $\xi$ ) is the product of two factors: the divergence of $\partial \bar{D}^{*}$, i.e., Laplacian of $\bar{D}^{*}$ (denoted as $\nabla^{2} \bar{D}^{*}$ ) and the absolute component difference of $\partial \bar{D}^{*}$ (denoted as diff $\partial \bar{D}^{*}$ ). If we focus on a 2dimensional (2D) system for simplicity, the second factor can be expressed as $\sqrt{\left|\bar{D}_{x}^{* 2}-\bar{D}_{y}^{* 2}\right|}$, where $\bar{D}_{x}^{*}$ and $\bar{D}_{y}^{*}$ represent the $x$ and $y$ component of $\partial \bar{D}^{*}$, respectively. Therefore, for the $2 \mathrm{D}$ system, the dimensionless parameter $\xi$ can be calculated as

$\xi=\nabla^{2} \bar{D}^{*} \cdot \sqrt{\left|\bar{D}_{x}^{* 2}-\bar{D}_{y}^{* 2}\right|} \cdot v_{\text {aver }}$,

where $v_{\text {aver }} \sim r_{\text {aver }}^{3}$ is the volume on average.

\subsection{Correlation between $\xi$ and STZs}

The correlation between $\xi$ and STZs is presented in Fig. 2. The contoured plot in Fig. 2(a) shows the distribution of $\xi$ which is calculated based on the atomic configuration at the initial state $(\gamma=0)$. The dark circles represent STZ atoms selected based on the change of $\eta_{i}{ }^{\text {Mises }}$ as done in the other work (Şopu et al., 2017; Tian et al., 2017; Xu et al., 2018). At a specific $\gamma$, atoms with $\Delta \eta_{i}{ }^{\operatorname{Mises}}(\gamma)=\eta_{i}{ }^{\operatorname{Mises}}(\gamma)-\eta_{i}{ }^{\text {Mises }}(\gamma-\Delta \gamma)>\Delta \eta_{t}$ are deemed to be STZ atoms, where $\Delta \gamma=0.1 \%, \Delta \eta_{t}=0.005$ which is the lowest $\Delta \eta^{\text {Mises }}$ of the first plastic event in Fig. 1(b). Since STZ appears step by step at different shear strain, Fig. 2(a) shows the distribution of STZ atoms from $\gamma=0.1 \%$ to $9.0 \%$. The $9.0 \%$ is chosen because most of the samples start to yield at this strain, which is apparent in the stress-strain curve in Fig. 1(b). Obviously, most of the STZ atoms are located where $\xi$ is positive and relatively large. Fig. 2(b) shows the correlation between $\xi$ and STZs in a more quantitative way. There are three atom sets defined in Fig. 2(b):

(i) Set $S_{\gamma}$ contains STZ atoms appearing at $\gamma$, i.e., atoms with $\Delta \eta_{i}^{\text {Mises }}(\gamma)>\Delta \eta_{t}$ during interval $[\gamma-\Delta \gamma, \gamma]$;

(ii) Set $S$ contains all the STZ atoms that have experienced shear transformation before $\gamma=9.0 \%$. Therefore, all the atoms in $S$ can be determined by $S=\cup_{\gamma=0.1 \%}^{9.0 \%} S_{\gamma}$;

(iii) Set $P$ contains atoms with $\xi_{i}>\xi_{t}$, where the threshold value $\xi_{t}=2 \times 10^{-4}$ is selected based on the distribution of $\xi$, i.e., atoms in set $P$ are predicted to be near regions of preexisted defects.

The data bar at $\gamma$ in Fig. 2(b) shows the value of $N_{S_{\gamma} \cap P} / N_{S_{\gamma}}$, i.e. how many atoms in $S_{\gamma}$ is successfully predicted, where $N_{S_{\gamma}}$ is the 


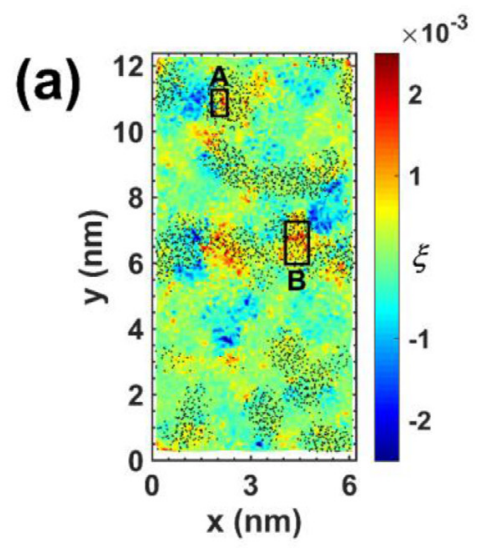

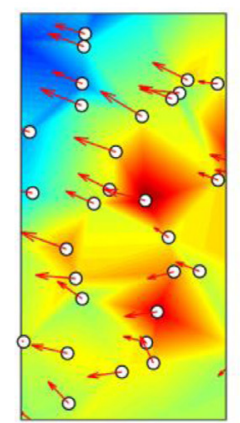

A

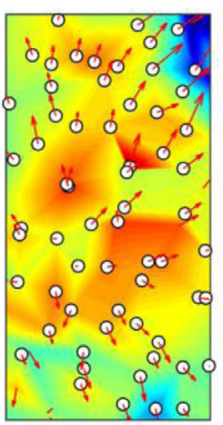

B
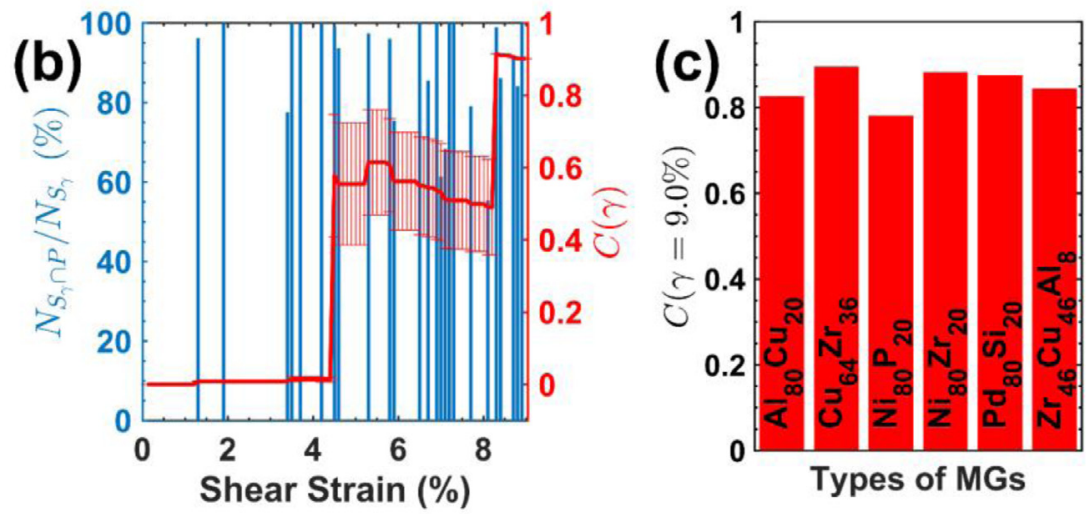

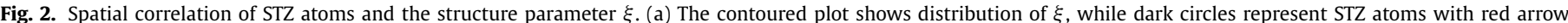

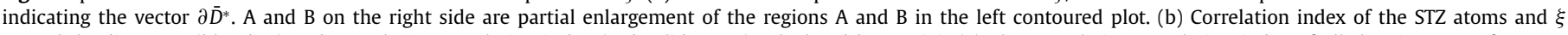

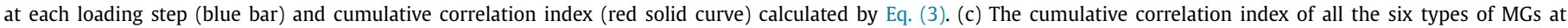
$\gamma=0.9 \%$. For each type of MG, the cumulative correlation index is the averaged among five different samples.

number of atoms in the set $S_{\gamma} . S_{\gamma} \cap P$ is the intersection of atom sets $S_{\gamma}$ and $P$. There is no data bar in Fig. 2(b) at some loading steps means that no STZ is activated during the strain interval of $[\gamma-\Delta \gamma, \gamma]$. The results show that at the strain of $9.0 \%$, there are $89.8 \%$ of atoms in $S_{\gamma}$ which are also in the predicted set $P$. The solid curve in Fig. 2(b) depicts the correlation parameter $C(\gamma)$ employed by Manning and Liu (2011) in the correlation analysis of soft modes:

$C(\gamma)=\frac{\hat{S}(\gamma) \cdot \hat{P}}{N_{P}}+\frac{[I-\hat{S}(\gamma)] \cdot[I-\hat{P}]}{N-N_{P}}-1$,

where $N$ is the number of total atoms in the sample. $\hat{S}(\gamma)$ and $\hat{P}$ are binary vectors. $\hat{S}(\gamma)=\left\{\hat{S}_{i}(\gamma) \in\{0,1\}\right\}$, where $\hat{S}_{i}(\gamma)=1$ if atom $i$ is experiencing shear transform during interval $[\gamma-\Delta \gamma, \gamma]$. $\hat{P}=\left\{\hat{P}_{i} \in\{0,1\}\right\}$, where $\hat{P}_{i}=1$ if atom $i$ has $\xi>\xi_{t} . C(\gamma)=1.0$ only if $\hat{S}(\gamma)$ and $\hat{P}$ are perfectly matched, while $C(\gamma)=0.0$ if $\hat{S}(\gamma)$ and $\hat{P}$ are uncorrelated. When $\gamma<5.0 \%$, only a few STZ atoms have been activated, so $C(\gamma)$ is reasonably much less than 1.0. However, as there are more and more STZ atoms, $C(\gamma)$ approaches 1.0. Particularly, $C(\gamma)$ at $\gamma=0.9 \%$ is 0.9 , indicating that around $90 \%$ of the STZ activation occurs in the atom set $P$ before shear band formation. We have simulated several other types of MGs with results shown in Fig. 2(c), where all $C(\gamma)$ at $\gamma=0.9 \%$ are around 0.8 . Therefore, the parameter $\xi$ is valid for prediction of activation of STZs in MGs with diverse types of element.

\subsection{Relationship between $\partial \bar{D}^{*}$ and nonaffine displacement}

According to the definition of $\xi$ in Eq. (3) and the simulation results, the regions with positively large $\nabla^{2} \bar{D}^{*}$ and $\operatorname{diff} \partial \bar{D}^{*}$ are the most possible locations where STZ will be activated during shear loadings. Recall that $\bar{D}^{*}$ is statistically proportional to local equivalent stiffness, therefore, atoms with positive $\nabla^{2} \bar{D}^{*}$ are corresponding to localized soft regions, i.e. the center of positive $\nabla^{2} \bar{D}^{*}$ region is relatively softer than peripheral regions. The relationship between soft regions and STZ activation is similar to the conclusion of some other work based on experiments and simulations of MGs. For example, Şopu et al. suggested a model of shear band composed of sequential soft STZs and hard icosahedral clusters (Şopu et al., 2017). Recently, Wang et al. proposed a localized "gradient atomic packing structure" comprising solid-like, transition, and liquid-like region (Wang et al., 2018a, 2018b). However, localized soft region is a necessary but not sufficient condition for STZ activation. The type of external loading (Gendelman et al., 2015) and the distribution shape of $\partial \bar{D}^{*}$ also affect the formation of STZ, so $\operatorname{diff} \partial \bar{D}^{*}$ is introduced in the parameter $\xi$.

Mechanism behind $\xi$ is the relationship among local heterogeneity, atomic nonaffine displacements and localized shear deformation in MGs. Firstly, how the $\partial \bar{D}^{*}$ is related to atomic nonaffine displacements is demonstrated in Fig. 3. In a 2D equilibrium MG system as shown in Fig. 3(a), the force on an atom is equilibrated in different directions by interactions of neighbor atoms. However, the equivalent stiffness around the atom could be unequal in different directions due to the random distribution of neighbors in MG system. Local dynamical matrix $D_{i}$ in Eq. (2) represents the anisotropy of local equivalent stiffness of the atom. For simplicity, suppose the axes 1 and 2 in Fig. 3(a) overlap the two principal directions of external loading strain; $\varepsilon_{1}$ and $\varepsilon_{2}$ are the two corresponding principal strain. $k_{1}, k_{1+}$, $k_{2}$, and $k_{2}+$ are used to represent different local equivalent 

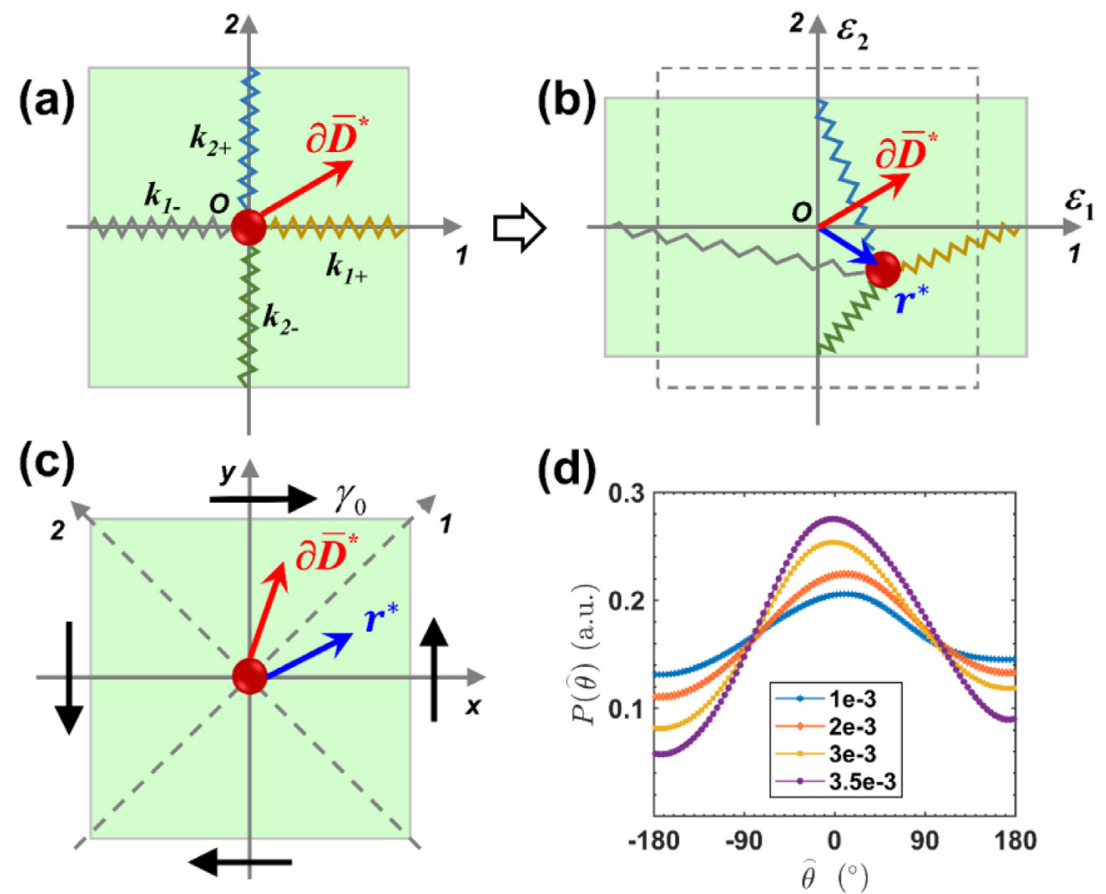

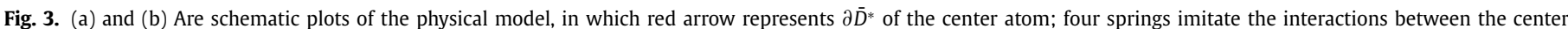

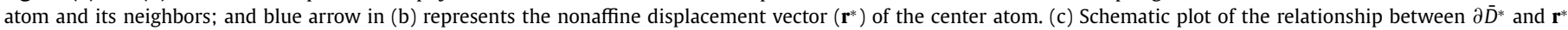

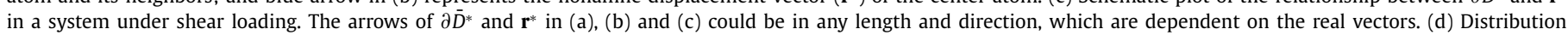

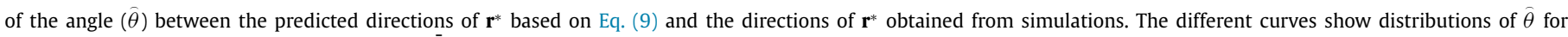
various threshold values of the magnitude of $\partial \bar{D}^{*}$.

stiffness in different directions due to the break of centrosymmetry (Zaccone, 2013; Zaccone and Scossa-Romano, 2011). The springs in Fig. 3(a) are used to represent the simplified inhomogeneous stiffness environment contributed by all the neighbors of an atom instead of just the nearest-neighbor bonds, so the boundary of the rectangular box in Fig. 3(a) does not represent the symmetric positions of nearest-neighbors but an arbitrary local region around an atom. Zaccone et al. have presented a mathematical deduction of nonaffine displacements under elastic heterogeneity and non-centrosymmetry (Zaccone, 2013; Zaccone and ScossaRomano, 2011), which is applied here to analysis the simplified 2D physical model. According to the definition of affine force $\underline{\underline{\Xi}}$ (Zaccone and Scossa-Romano, 2011)

$\Xi_{j k}=\left.D_{i j} \frac{\mathrm{d} r_{i}^{*}}{\mathrm{~d} \varepsilon_{k}}\right|_{\varepsilon_{k}=0}$,

where the nonaffine displacement $\mathbf{r}^{*}$ can be expressed under the first-order approximation by

$r_{j}^{*}=\frac{\Xi_{j} \varepsilon_{j}}{D_{j}}$,

where $D_{i j}=0$ and $\Xi_{i j}=0$ when $i \neq j$ under coordinates of the principal direction, so $D_{j j}$ is abbreviated as $D_{j}$ and $\Xi_{j j}$ is abbreviated as $\Xi_{j}$. Here $D_{j}=k_{j+}+k_{j-}$ is the component of local dynamical matrix. Then $\Xi_{j}$ can be also written as (Zaccone, 2013)

$\Xi_{j}=-\left.\left(\frac{\partial^{2} V}{\partial r_{j}^{*} \partial r_{j}{ }^{0}}\right)\right|_{r_{j}^{*}} \frac{\partial r_{j}{ }^{0}}{\partial \varepsilon_{j}}=a\left(k_{j+}-k_{j-}\right)$,

where $r_{j}{ }^{0}$ is the affine displacement, $j=1,2$ indicates the direction and $a$ is the length of the local region. As a result, when the system is subjected to external strain $\varepsilon_{1}$ and $\varepsilon_{2}$, the atomic nonaffine displacement $\mathbf{r}^{*}$ can be calculated as

$r_{j}^{*}=\varepsilon_{j} a \frac{k_{j+}-k_{j-}}{k_{j+}+k_{j-}}$, where $\frac{k_{j+}-k_{j-}}{k_{j+}+k_{j-}}$ represents the variation of equivalent stiffness in the direction $j$. Recall that the $\bar{D}^{*}$ field reflects the distribution of stiffness in the atomic system, then the $j$ thcomponent of $\partial \bar{D}^{*}$ should be proportional to $\frac{k_{j+}-k_{j-}}{k_{j+}+k_{j-}}$, i.e., $\frac{k_{j_{+}}-k_{j-}}{k_{j+}+k_{j-}} \sim \frac{a}{\bar{D}_{j}} \frac{\mathrm{d} D_{j}}{\mathrm{~d} x_{j}} \sim a \partial \bar{D}_{j}^{*}$. As a result, the nonaffine displacement $\mathbf{r}^{*}$ is related to $\partial \bar{D}^{*}$ as

$r_{j}^{*} \sim \varepsilon_{j} a^{2} \partial \bar{D}_{j}^{*}$

For the special loading case of pure shear with the principal strain of $\varepsilon_{1}=\gamma_{0}$ and $\varepsilon_{2}=-\gamma_{0}$, according to Eq. (9), the direction of $\mathbf{r}^{*}$ and $\partial \bar{D}^{*}$ is symmetric about axis 1 or the axis $y=x$ as shown in Fig. 3(c). To confirm this conclusion, distribution of the angle (denoted by $\hat{\theta}$ ) between the predicted directions of $\mathbf{r}^{*}$ based on Eq. (9) and the directions of $\mathbf{r}^{*}$ obtained from simulations is presented in Fig. 3(d). The distribution peak located at zero indicates that most of the predicted directions of $\mathbf{r}^{*}$ are coincident with that from simulations. The different distribution curves in Fig. 3(d) are obtained from atoms selected by various threshold values of the magnitude of $\partial \bar{D}^{*}\left(\left|\partial \bar{D}^{*}\right|\right)$. Apparently, the larger $\left|\partial \bar{D}^{*}\right|$ is, the better Eq. (9) predicts, that's because larger $\left|\partial \bar{D}^{*}\right|$ implies higher local heterogeneity or non-centrosymmetry.

\subsection{Mechanism of $\xi$}

Once the relationship between $\mathbf{r}^{*}$ and $\partial \bar{D}^{*}$ is established by Eq. (9), why regions with positive and large $\xi$ will lead to activation of STZs can be explained. For the first factor of $\xi$, i.e., $\nabla^{2} \bar{D}^{*}$, if we consider $\bar{D}^{*}$ as a kind of stiffness potential field, then the landscape of the potential around a region with positive and relatively large value of $\nabla^{2} \bar{D}^{*}$ is similar to a valley with vectors of $\partial \bar{D}^{*}$ pointing against the center of the region. The larger $\nabla^{2} \bar{D}^{*}$ is, the steeper the valley will be. While $\nabla^{2} \bar{D}^{*}$ controls the steepness of the valley, the second factor of $\xi$, i.e., $\sqrt{\left|\bar{D}_{x}^{* 2}-\bar{D}_{y}^{* 2}\right|}$, controls the shape 

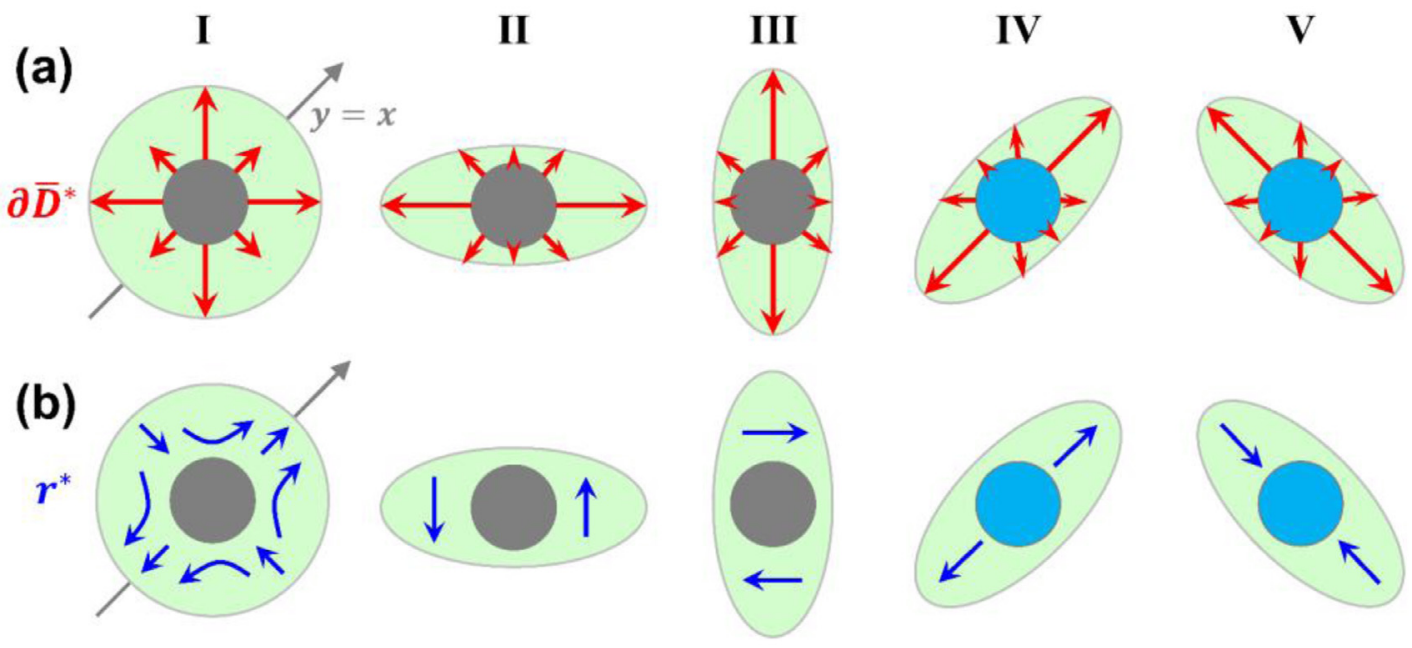

Fig. 4. (a) Distribution of $\partial \bar{D}^{*}$ in different modes. (b) Corresponding nonaffine displacement fields ( $\mathbf{r}^{*}$ ) for different distribution modes of $\partial \bar{D}^{*}$.

of the valley. Specifically, the region with larger $\sqrt{\left|\bar{D}_{x}^{* 2}-\bar{D}_{y}^{* 2}\right|}$ contains vectors $\partial \bar{D}^{*}$ with relatively larger length and direction along near either $x$ or $y$ axes. Five different distributions of $\partial \bar{D}^{*}$ in regions with positive $\nabla^{2} \bar{D}^{*}$ are illustrated in Fig. 4(a). The distributions are related to different nonaffine deformation modes. In the modes I, II and III, $\sqrt{\left|\bar{D}_{x}^{* 2}-\bar{D}_{y}^{* 2}\right|}$ is relatively large, so the major $\partial \bar{D}^{*}$ points along near either $x$ or $y$ axes; while in the modes IV and $\mathrm{V}$ with relatively small $\sqrt{\left|\bar{D}_{x}^{* 2}-\bar{D}_{y}^{* 2}\right|}$, the major $\partial \bar{D}^{*}$ points along near axes $y=x$ or $y=-x$. Based on the relationship between $\mathbf{r}^{*}$ and $\partial \bar{D}^{*}$ as discussed above, nonaffine displacement fields for the five modes can be predicted as shown in Fig. 4(b). Specifically, in the mode I, $\partial \bar{D}^{*}$ along $x$ and $y$ axes is relatively large, so $\mathbf{r}^{*}$ shows quadrupolar-style field which will strengthen shear deformation in this region. Actually, the quadrupolar-style nonaffine displacement fields have been observed in several previous work (Bulatov and Argon, 1994; Hieronymus-Schmidt et al., 2017; Maloney and Lemaitre, 2006) in which its connection with STZs has been discussed. In the modes II and III with major $\partial \bar{D}^{*}$ along $x$ and $y$ direction, respectively, the nonaffine displacement field imposes shear-like loading to the region which also strengthens shear deformation in the local region. Similar modes can be found in the simulation results as shown in Fig. 2(a) with A and B regions matching the modes II and III, respectively. While in the modes IV and V, the nonaffine displacement field around the local region corresponds to tension and compression deformation pattern respectively, and no shear deformation is strengthened. Therefore, STZs are more likely formed in mode I, II and III for local regions with positive and relatively large $\xi$. Recently, Şopu et al. also discovered that the STZ activation mechanism resembles the modes in shear band multiplication (Şopu et al., 2020).

Although the analysis is focused on a quasi-2D MG system under shear deformation, the philosophy of the parameter $\xi$ can be extended to identify similar defects in MGs under complex conditions. Here we refer the "defect" in MGs to atomic-level structural unit in the initial configuration which will lead to activation of STZs under specific external loadings. The two factors of $\xi$ are corresponding to two steps in the defect identification. Firstly, regions with positive and relatively large $\nabla^{2} \bar{D}^{*}$ are potential defects. Physically, the "stiffness valleys" identified by $\nabla^{2} \bar{D}^{*}$ are metastable regions in which localized STZ might be triggered by external loading. The mechanism of $\nabla^{2} \bar{D}^{*}$ is independent on external loading, so the first step is suitable for analysis in general cases. However, the second factor is loading-dependent. Under a given loading condition, only "stiffness valleys" with specific shapes will lead to localization or formation of STZs. Therefore, for loading conditions other than shear, the second factor of $\xi$ would be different. Additional analysis based on the relationship between $\mathbf{r}^{*}$ and $\partial \bar{D}^{*}$ should be performed to determine the form of the second factor. It is worth noting that as the sample deformed, STZ events would change the local configuration around STZs, so the local equivalent stiffness distribution and loading condition will change accordingly. To accommodate these changes, one should track the variation of configuration step by step and perform more general analysis for different loading conditions. Moreover, usage of the parameter can be extended to systems at finite temperature. In general, once the temperature is given, $\bar{D}^{*}$ is primarily dependent on atomic configuration, and its derivatives are independent on temperature. Therefore, in MD simulation at finite temperature, the initial configuration should be time averaged before calculating $\bar{D}^{*}$.

\section{Summary}

In summary, molecular simulations of MG samples under AQS shear loading condition are carried out to investigate the atomiclevel origin of STZs. It is found that a structural parameter $\xi$ in initial configuration of MGs shows strong correlation to the formation of STZs in the samples. Quantitative analysis indicates that more than $80 \%$ of the STZ atoms can be effectively predicted by $\xi$ in MG samples with different types of element. The parameter consists of two important factors which are the divergence and the absolute component difference of $\partial \bar{D}^{*}$, where $\partial \bar{D}^{*}$ is a vector representing the gradient of local equivalent stiffness. Physically, the first factor of $\xi$ (i.e. $\nabla^{2} \bar{D}^{*}$ ) with positive and relatively large value represents the "stiffness valleys" in the initial configuration which are potential defects; while the second factor of $\xi$ reveals the shape of the stiffness valleys. Results show that only regions with specific shapes of stiffness valleys will lead to the formation of STZs in MGs under shear loading. The mechanism behind the parameter $\xi$ is explained based on the analytical derivation of the relationship between atomic nonaffine displacement and $\partial \bar{D}^{*}$. The parameter $\xi$ not only reveals the relationship among local heterogeneity, nonaffine displacement, and shear localization, but also provides an efficient way for predicting activation of STZs in MGs. Moreover, the ideology of $\xi$ using Laplacian of local equivalent stiffness would bridge the analysis between atomic simulations and traditional mechanical theory for defects identification in MGs. 


\section{Declaration of Competing Interests}

The authors declare that they have no known competing financial interests or personal relationships that could have appeared to influence the work reported in this paper.

\section{CRediT authorship contribution statement}

Ronghao Shi: Software, Visualization, Investigation, Data curation, Writing - original draft. Pan Xiao: Conceptualization, Methodology, Software, Writing - review \& editing. Rong Yang: Methodology, Formal analysis. Yilong Bai: Supervision, Methodology.

\section{Acknowledgments}

Supports from the National Natural Science Foundation of China (Grant nos. 11790292, 11672298 and 11432014), the Strategic Priority Research Program (B) of the Chinese Academy of Sciences (XDB22040501) is gratefully acknowledged. Computations are performed on the ScGrid of Supercomputing Center, Computer Network Information Center of Chinese Academy of Sciences and LNMGrid of the State Key Laboratory of Nonlinear Mechanics.

\section{References}

Argon, A.S., 1979. Plastic deformation in metallic glasses. Acta Metall. 27, 47-58. doi:10.1016/0001-6160(79)90055-5.

Bulatov, V.V., Argon, A.S., 1994. A stochastic model for continuum elasto-plastic behavior. I. Numerical approach and strain localization. Model. Simul. Mater. Sci. Eng. 2, 167-184. doi:10.1088/0965-0393/2/2/001.

Cheng, Y.Q., Ma, E., Sheng, H.W., 2009. Atomic level structure in multicomponent bulk metallic glass. Phys. Rev. Lett. 102. doi:10.1103/PhysRevLett.102.245501.

Cubuk, E.D., Schoenholz, S.S., Kaxiras, E., Liu, A.J., 2016. Structural properties of defects in glassy liquids. J. Phys. Chem. B 120, 6139-6146. doi:10.1021/acs.jpcb. 6b02144.

DiDonna, B.A., Lubensky, T.C., 2005. Nonaffine correlations in random elastic media. Phys. Rev. E 72, 066619. doi:10.1103/PhysRevE.72.066619.

Ding, J., Cheng, Y.Q., Sheng, H., Asta, M., Ritchie, R.O., Ma, E., 2016. Universal structural parameter to quantitatively predict metallic glass properties. Nat. Commun. 7, 13733. doi:10.1038/ncomms13733.

Ding, J., Patinet, S., Falk, M.L., Cheng, Y., Ma, E., 2014a. Soft spots and their structural signature in a metallic glass. Proc. Natl. Acad. Sci. 111, 14052-14056. doi:10.1073/pnas.1412095111.

Ding, J., Xu, M., Guan, P.F., Deng, S.W., Cheng, Y.Q., Ma, E., 2014b. Temperature effects on atomic pair distribution functions of melts. J. Chem. Phys. 140, 064501. doi:10.1063/1.4864106.

Fultz, B., 2010. Vibrational thermodynamics of materials. Prog. Mater. Sci. doi:10. 1016/j.pmatsci.2009.05.002.

Gendelman, O., Jaiswal, P.K., Procaccia, I., Sen Gupta, B., Zylberg, J., 2015. Shear transformation zones: state determined or protocol dependent? epl 109, 16002. doi:10.1209/0295-5075/109/16002.

Greer, A.L., Cheng, Y.Q., Ma, E., 2013. Shear bands in metallic glasses. Mater. Sci. Eng. R Rep. 74, 71-132. doi:10.1016/j.mser.2013.04.001.

Hieronymus-Schmidt, V., Rösner, H., Wilde, G., Zaccone, A., 2017. Shear banding in metallic glasses described by alignments of Eshelby quadrupoles. Phys. Rev. B 95, 134111. doi:10.1103/PhysRevB.95.134111.

Hu, Y.C., Guan, P.F., Li, M.Z., Liu, C.T., Yang, Y., Bai, H.Y., Wang, W.H., 2016. Unveiling atomic-scale features of inherent heterogeneity in metallic glass by molecular dynamics simulations. Phys. Rev. B 93, 214202. doi:10.1103/PhysRevB.93.214202.

Jiang, F., Jiang, M.Q., Wang, H.F., Zhao, Y.L., He, L., Sun, J., 2010. Shear transformation zone volume determining ductile-brittle transition of bulk metallic glasses. doi:10.1016/j.actamat.2010.12.006.

Jiang, M.Q., Dai, L.H., 2010. Short-range-order effects on intrinsic plasticity of metallic glasses. Philos. Mag. Lett. 90, 269-277. doi:10.1080/09500831003630781.

LeSar, R., Najafadabi, R., Srolovitz, D.J., 1989. Finite-temperature defect properties from free energy minimization. Phys. Rev. Lett. 63, 624.

Maloney, C.E., Lemaitre, A., 2006. Amorphous systems in athermal, quasistatic shear. Phys. Rev. E 74, 016118. doi:10.1103/PhysRevE. 74.016118.

Manning, M.L., Liu, A.J., 2011. Vibrational modes identify soft spots in a sheared disordered packing. Phys. Rev. Lett. 107, 108302. doi:10.1103/PhysRevLett.107. 108302.
Milkus, R., Zaccone, A., 2016. Local inversion-symmetry breaking controls the boson peak in glasses and crystals. Phys. Rev. B 93, 094204. doi:10.1103/PhysRevB.93. 094204.

Palyulin, V.V., Ness, C., Milkus, R., Elder, R.M., Sirk, T.W., Zaccone, A., 2018. Parameter-free predictions of the viscoelastic response of glassy polymers from non-affine lattice dynamics. Soft Matter 14, 8475-8482. doi:10.1039/ c8sm01468j.

Peng, C.X., Şopu, D., Song, K.K., Zhang, Z.T., Wang, L., Eckert, J., 2017. Bond length deviation in Cuzr metallic glasses. Phys. Rev. B 96, 174112. doi:10.1103/PhysRevB. 96.174112.

Plimpton, S., 1995. Fast parallel algorithms for short-range molecular dynamics. J. Comput. Phys. 117, 1-19. doi:10.1006/jcph.1995.1039.

Rottler, J., Schoenholz, S.S., Liu, A.J., 2014. Predicting plasticity with soft vibrational modes: from dislocations to glasses. Phys. Rev. E 89, 42304. doi:10.1103/ PhysRevE.89.042304.

Schuh, C.A., Hufnagel, T.C., Ramamurty, U., 2007. Mechanical behavior of amorphous alloys. Acta Mater. 55, 4067-4109. doi:10.1016/j.actamat.2007.01.052.

Shimizu, F., Ogata, S., Li, J., 2007. Theory of shear banding in metallic glasses and molecular dynamics calculations. Mater. Trans. 48, 2923-2927. doi:10.2320/ matertrans.MJ200769.

Sopu, D., Scudino, S., Bian, X.L., Gammer, C., Eckert, J., 2020. Atomic-scale origin of shear band multiplication in heterogeneous metallic glasses. Scr. Mater. 178, 57-61. doi:10.1016/j.scriptamat.2019.11.006.

Sopu, D., Stukowski, A., Stoica, M., Scudino, S., 2017. Atomic-Level processes of shear band nucleation in metallic glasses. Phys. Rev. Lett. 119, 195503. doi:10.1103/ PhysRevLett.119.195503.

Spaepen, F., 1977. A microscopic mechanism for steady state inhomogeneous flow in metallic glasses. Acta Metall. 25, 407-415. doi:10.1016/0001-6160(77)90232-2.

Stukowski, A., 2010. Visualization and analysis of atomistic simulation data with OVITO-the open visualization tool. Model. Simul. Mater. Sci. Eng. 18, 7. doi:10. 1088/0965-0393/18/1/015012.

Taylor, G.I., 1934. The mechanism of plastic deformation of crystals. Part I. Theoretical. Proc. R. Soc. A Math. Phys. Eng. Sci. 145, 362-387. doi:10.1098/rspa.1934. 0106.

Tian, Z.L., Wang, Y.J., Chen, Y., Dai, L.H., 2017. Strain gradient drives shear banding in metallic glasses. Phys. Rev. B 96, 094103. doi:10.1103/PhysRevB.96.094103.

Wang, B., Luo, L., Guo, E., Su, Y., Wang, M., Ritchie, R.O., Dong, F., Wang, L. Guo, J., Fu, H., 2018a. Nanometer-scale gradient atomic packing structure surrounding soft spots in metallic glasses. npj. Comput. Mater 4, 41. doi:10.1038/ s41524-018-0097-4.

Wang, H., Hu, M., Xia, M., Ke, F., Bai, Y., 2008. Molecular/cluster statistical thermodynamics methods to simulate quasi-static deformations at finite temperature. Int. J. Solids Struct. 45, 3918-3933. doi:10.1016/j.ijsolstr.2007.12.023.

Wang, N., Ding, J., Luo, P., Liu, Y., Li, L., Yan, F., 2018b. Chemical variation induced nanoscale spatial heterogeneity in metallic glasses. Mater. Res. Lett. 6, 655-661. doi:10.1080/21663831.2018.1532465.

Wang, Z., Wang, W.H., 2019. Flow units as dynamic defects in metallic glassy materials. Natl. Sci. Rev. 6, 304-323. doi:10.1093/nsr/nwy084.

Wei, D., Yang, J., Jiang, M.Q., Wei, B.C., Wang, Y.J., Dai, L.H., 2019. Revisiting the structure-property relationship of metallic glasses: common spatial correlation revealed as a hidden rule. Phys. Rev. B 99, 014115. doi:10.1103/PhysRevB.99. 014115.

Widmer-Cooper, A., Perry, H., Harrowell, P., Reichman, D.R., 2008. Irreversible reorganization in a supercooled liquid originates from localized soft modes. Nat. Phys. 4, 711-715. doi:10.1038/nphys1025.

Xiao, P., Wang, J., Ke, F.J., Bai, Y.L., 2012. Molecular statistical thermodynamics - a distinct and efficient numerical approach to quasi-static analysis of nanomaterials at finite temperature. Compos. B: Eng. 57-63. doi:10.1016/j.compositesb. 2011.04.031.

Xu, B., Falk, M.L., Li, J.F., Kong, L.T., 2018. Predicting shear transformation events in metallic glasses. Phys. Rev. Lett. 120, 125503. doi:10.1103/PhysRevLett.120. 125503.

Yang, J., Wang, Y.J., Ma, E., Zaccone, A., Dai, L.H., Jiang, M.Q., 2019. Structural parameter of orientational order to predict the boson vibrational anomaly in glasses. Phys. Rev. Lett. 122, 015501. doi:10.1103/PhysRevLett.122.015501.

Yang, X., Liu, R., Yang, M. Wang, W.H., Chen, K., 2016. Structures of local rearrangements in soft colloidal glasses. Phys. Rev. Lett. 116, 238003. doi:10.1103/ PhysRevLett.116.238003.

Zaccone, A., 2013. Elastic deformations in covalent amorphous solids. Mod. Phys. Lett. B 27, 1330002, doi:10.1142/S0217984913300020.

Zaccone, A., Scossa-Romano, E., 2011. Approximate analytical description of the nonaffine response of amorphous solids. Phys. Rev. B 83, 184205. doi:10.1103 PhysRevB.83.184205.

Zhang, W., Douglas, J.F., Starr, F.W., 2019. What does the instantaneous normal mode spectrum tell us about dynamical heterogeneity in glass-forming fluids? J. Chem. Phys. 151, 184904. doi:10.1063/1.5127821.

Zink, M., Samwer, K., Johnson, W.L., Mayr, S.G., 2006. Plastic deformation of metallic glasses: size of shear transformation zones from molecular dynamics simulations. Phys. Rev. B 73, 172203. doi:10.1103/PhysRevB.73.172203. 